\title{
Can stacking faults in hard-sphere crystals anneal out spontaneously?
}

\author{
Sander Pronk and Daan Frenkel ${ }^{\mathrm{a})}$ \\ FOM Institute for Atomic and Molecular Physics, Kruislaan 407, 1098 SJ Amsterdam, The Netherlands
}

(Received 17 September 1998; accepted 30 November 1998)

\begin{abstract}
We estimate the rate at which randomly stacked hard-sphere crystals transform into the thermodynamically stable face-centered cubic phase. As an input for this estimate we need both the free-energy difference between bulk face-centered cubic (fcc) and hexagonal close packed (hcp) phases, and the hcp-fcc interfacial free energy. The latter quantity was computed using a lattice-switch Monte Carlo (MC) simulation method. We find the interfacial free energy to be nonzero but extremely small: $26 \pm 6 \cdot 10^{-5} k T / \sigma^{2}$, where $\sigma$ is the particle diameter. The free energy difference between the bulk phases was calculated using two different techniques. On the basis of our simulation results we estimate that in hard-sphere colloidal suspensions millimeter-sized randomly stacked crystal will anneal to form essentially pure fcc crystal on a time scale of months to years. (C) 1999 American Institute of Physics. [S0021-9606(99)51709-1]
\end{abstract}

\section{INTRODUCTION}

The freezing of hard spheres is one of the most dramatic illustrations that the emergence of crystalline order can be entropy driven. Ever since the early simulations of Alder, Wainwright ${ }^{1}$ and Wood and Jacobson, ${ }^{2}$ hard-sphere freezing has been studied extensively, both theoretically ${ }^{3-6}$ and experimentally. ${ }^{7,8}$ Hard spheres can occur in two different crystal structures, face-centered cubic (fcc) and hexagonal close packed (hcp). These two phases differ in the stacking of the hexagonal close-packed [111] layers. The fcc phase has $A B C A B C \cdots$ stacking, while the hcp phase has $A B A B A B \cdots$ stacking. Of the two crystal structures, the fcc phase is the most stable. Recent simulations suggest that, at the melting density $\left(\rho / \rho_{0} \approx 0.736\right.$, where $\rho_{0}$ is the density at regular close packing) the fcc phase is more stable than hcp by an amount of the order of $9 \pm 2 \cdot 10^{-4} k_{B} T$ per particle. ${ }^{9,10}$ As the free-energy difference between the two phases is very small, the spontaneous generation of stacking faults is quite common. In fact, recent experiments on the crystallization under micro-gravity conditions of suspensions of harshly repulsive colloidal spheres (henceforth referred to as "hardsphere colloids"), found that randomly stacked hexagonal close-packed (rhcp) crystallites were formed. ${ }^{11}$ Yet, there is experimental evidence that, in slowly grown crystallites, the fcc phase is favored over the hcp phase, and fcc stacking of hexagonal close-packed planes occurs with a higher than random probability. ${ }^{12-14}$ The aim of the present paper is to estimate the driving force for the formation of a pure fcc phase from the randomly stacked phase. Using some simple assumptions about the rate of crystal growth, we can then arrive at an estimate of the growth velocity of essentially pure fcc crystals from a poly-crystalline mixture of randomly stacked crystals. Our main conclusion is that the driving force, although weak, is large enough to account for a growth rate of fcc crystals that is of the order of Angstrøms per second, for colloids with a hard-core diameter in the 100

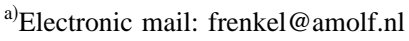

nm range. In other words, for a typical hard-sphere colloid, it would take months to transform a one-millimeter crystallite from rhcp to fcc. For a 50-100 $\mu$ crystallite, the time would be days, rather than months. However, fcc crystallites that are found to form much faster, cannot be pure hard-sphere crystals.

To estimate the rate at which the fcc phase grows from the rhcp phase, we need to estimate the relative free energy of the latter. The free energy of the rhcp phase contains several ingredients: First of all, $\Delta f=f_{\mathrm{hcp}}-f_{\text {fcc }}$, the difference in bulk free energies per particle of the pure fcc and hcp phases. Secondly, the interfacial free energy $\gamma_{\mathrm{hcp}-\mathrm{fcc}}$, which is the measure of the additional free-energy cost to create an fcc-hcp interface. And thirdly, the stacking entropy of the rhcp phase $\left(k_{B} \ln 2\right.$ per plane). Although $\Delta f$ is known from recent simulations,,${ }^{9,10}$ its value has been subject to debate. ${ }^{5,6,9,10} \mathrm{We}$, therefore, recomputed it using two different techniques. We find that the different approaches do indeed yield the same answer. To compute $\gamma_{\mathrm{hcp} \text {-fcc }}$ we used a lattice-switch Monte Carlo technique that is described in some detail below. Finally, to estimate the actual growth rate, we make use of the version of the Wilson-Frenkel law, ${ }^{15}$ as applied to colloids by several authors. ${ }^{3,4,7,8}$

\section{LATTICE-SWITCH MONTE CARLO}

To compute the free energy of the fcc-hcp interface, we used the lattice-switch method proposed by Bruce and Wilding. ${ }^{10}$ This method is particularly suited to compute the free-energy difference between two different solid structures, provided they have the same number of degrees of freedom. As a test, we used the same method to compute the freeenergy difference between the bulk fcc and hcp phases. In the lattice-switch simulations, we consider two realizations of the crystal structure that are related through a simple oneto-one particle mapping. The configuration of the system is denoted by an index $\alpha$. The particle positions in configuration $\alpha$ are denoted by $\vec{X}_{i}^{\alpha}+\vec{\delta}_{i}$, where $\vec{X}_{i}^{\alpha}$ is the lattice position for particle $i$ in configuration $\alpha$, and $\vec{\delta}_{i}$ is the displace- 


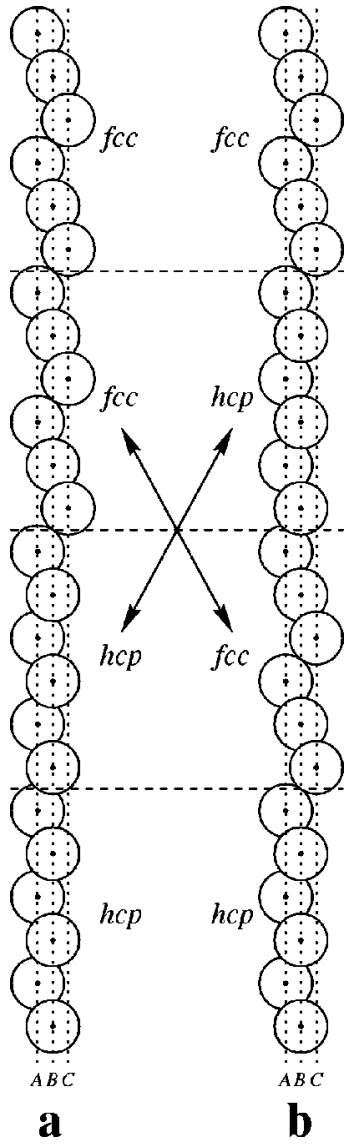

FIG. 1. To compute the fcc-hcp interfacial free energy, we used the latticeswitch Monte Carlo method to evaluate the free-energy difference between structures (a) (left) and (b) (right), shown in side view. Crystal a contains one fcc slab and an hcp slab of equal thickness. In structure (b), the total amount of fcc and hcp is the same, but now there are four crystal slabs. As a consequence, the total area of the fcc-hcp interface in (b) is twice that in (a).

ment relative to its lattice position. The different crystalline configurations differ in their lattice positions $\vec{X}_{i}^{\alpha}, i=1 \cdots N$. Below, we discuss two lattice switches. In the first, we consider a lattice switch from a pure fcc to a pure hcp phase. In the second, the initial configuration corresponds to a crystal structure in which the first half of the hexagonal layers have fcc stacking and the second half in hcp, while the final configuration has the middle two quarters swapped, creating a crystal which has four parts: fcc, hcp, fcc, and hcp (See Fig. 1). In this case, the net result is that the initial configuration has two interfaces between fcc and hcp, while the final one has four (with periodic boundary conditions). Yet, the total amount of fcc and hep both configurations is the same.

We can define a global partition function, $Z(N, V, T)$ as the sum of the partial partition functions for the different configurations $\alpha$

$Z(N, V, T)=\sum_{\alpha} Z(N, V, T, \alpha)=\sum_{\alpha} \int_{V} d \vec{\delta}_{i} \prod_{i} e^{-\Phi\left(\vec{\delta}_{i}, \alpha\right)}$,

where $\Phi$ represents the configurational energy in units $k_{B} T$. If the system can switch between different configurations by
TABLE I. Simulation results for the free energy difference between fcc and hcp structures of the hard-sphere crystal. The system size is denoted by $N$. EI stands for Einstein integration, and LS for the lattice-switch method. Except for the last line, all data correspond to a reduced density of $\rho / \rho_{0}$ $=0.7778$. The data in the bottom line are for $\rho / \rho_{0}=0.736$.

\begin{tabular}{rcl}
\hline \hline \multicolumn{1}{c}{$N$} & $\Delta f_{\text {hcp-fcc }}\left(10^{-5} k_{B} T\right)$ & \multicolumn{1}{c}{ Method } \\
\hline 216 & $101 \pm 4$ & LS (Ref. 10) \\
1728 & $83 \pm 3$ & LS (Ref. 10) \\
5832 & $86 \pm 3$ & LS (Ref. 10) \\
216 & $132 \pm 4$ & LS (this work) \\
1728 & $112 \pm 4$ & LS (this work) \\
1728 & $113 \pm 4$ & EI (this work) \\
12096 & $90 \pm 20$ & EI (Ref. 9) \\
\hline \hline
\end{tabular}

replacing the lattice positions $\vec{X}_{i}$ but retaining the displacements $\vec{\delta}_{i}$, the probability of finding the system in configuration $\alpha$ is

$$
P(\alpha \mid N, V, T)=\frac{Z(N, V, T, \alpha)}{Z(N, V, T)} .
$$

The Helmholtz free energy of configuration $\alpha$ is $f_{\alpha}$ $\equiv N^{-1} F_{\alpha} \equiv N^{-1} k T \ln Z(N, V, T, \alpha)$ and the free energy difference between the initial and final configurations (denoted by $\alpha_{i}$ and $\alpha_{f}$ ) can be written as

$$
\Delta f=\frac{k T}{N} \ln \frac{P\left(\alpha_{f} \mid N, V, T\right)}{P\left(\alpha_{i} \mid N, V, T\right)} .
$$

The probabilities $P(\alpha \mid N, V, T)$ are sampled using the histogram method of Ref. 10.

For the calculations on the free-energy difference between fcc and hcp structures we have used an fcc crystal as configuration $\alpha_{i}$ and an hcp crystal as configuration $\alpha_{f}$. To calculate the interfacial free energy between fcc and hcp we have used a system where the layer stacking for configuration $\alpha_{i}$ is the structure denoted in Fig. 1(a), while configuration $\alpha_{f}$ corresponds to Fig. 1(b) in the same figure. We obtain the hcp-fcc interfacial free energy density by computing the free energy difference between Figs. 1(a) and 1 (b), and dividing this difference by the difference in interfacial area of Figs. 1(a) and 1(b).

\section{RESULTS}

Most simulations were performed at a reduced density of $\rho / \rho_{0}=0.7778$, (packing fraction $\eta=0.5760$ ), a density somewhat higher than the melting density. This density was chosen because, at the melting density, mechanical instabilities can occur for intermediate values of the lattice-switch parameter $\alpha$-i.e., the various crystal stackings themselves are mechanically stable, but the (unphysical) states in between may exhibit a shear instability. Another reason to select the reduced density $\rho / \rho_{0}=0.7778$ is that it allowed us to compare our data with those of Ref. 10. In all simulations, periodic boundary conditions were used.

We first calculated the free energy difference per particle between fcc and hcp stackings $\left(\Delta f_{\text {hcp-fcc }}\right)$. In Table I we present our results. In the same table, we also show the free- 
energy difference at a reduced density of 0.736 (the melting density), as computed by Bolhuis et al. ${ }^{9}$ using the Einstein integration method. ${ }^{5}$ Our results differ by a small but statistically significant amount from those of Bruce and Wilding. ${ }^{10}$ This is troubling because the present simulations are only marginally longer than those of Ref. 10 (the longest runs in Ref. 10 sampled over $5 \times 10^{7}$ MC cycles-all our runs were about $30 \%$ longer). We, therefore, felt it necessary to check our results using a completely different numerical technique. To this end, we performed an Einstein integration on the 1728-particle system, using the same code that was employed for the calculations in Ref. 9.

The results of these simulations agreed to within the (small) statistical error with our lattice-switch MC results. In Ref. 10 it was suggested that the lattice-switch technique is substantially more precise than the Einstein integration method-we find that, for simulations of comparable length, the two methods are about equally accurate. We can use our results for $N=216$ and $N=1728$ to estimate $\Delta f_{\text {hcp-fcc }}$ in the limit $N \rightarrow \infty$. If we assume that finite-size corrections scale as $1 / N$, then the results for $N=1728$ are, to within the statistical error, equal to the results for the infinite system.

The calculation of the interfacial free energy was performed on a $12 \times 12 \times 24=3456$ particle system at a reduced density $\rho / \rho_{0}=0.7778$. The total length of the simulation was $6 \cdot 10^{8}$ Monte Carlo cycles. The hcp-fcc interfacial free energy was found to be $26 \pm 6 \cdot 10^{-5} k_{B} T / \sigma^{2}$, with $\sigma$ the particle diameter. In what follows, all free energies will be expressed in units of $k_{B} T$ and all distances in units of the particle diameter $\sigma$, unless otherwise noted.

\section{DISCUSSION}

Using the numerical data presented above, we can estimate the free-energy difference between the stable fcc phase and the rhcp phase. If we assume that the stacking in the rhcp phase is truly random, then the free-energy difference per particle is

$$
\Delta f_{\text {rhcp-fcc }}=0.5 \Delta f_{\text {hcp-fcc }}+0.25 \gamma_{\text {hcp-fcc }} s_{0}-\ln 2 / N_{\text {layer }},
$$

where $s_{0}$ is the surface area per particle $\left(s_{0}=(\sqrt{3} / 2)\right.$ $\times\left(\rho_{0} / \rho\right)^{2 / 3} \sigma^{2} \approx 1.02 \sigma^{2}$, for $\left.\rho / \rho_{0}=0.7778\right)$ and $N_{\text {layer }}$ is the number of particles in a single close-packed layer. In Eq. (4), we have assumed that the interfacial free energy does not depend on the density of stacking faults-in other words, we ignore the interaction between successive interfaces. Moreover, we assume that all stacking faults (which are fcc-hcp, fcc-fcc, or hcp-hcp) have the same interfacial free energy. This is, of course, an approximation. However, as the stacking-fault free energy itself is small, the resulting error is probably negligible.

In a randomly stacked crystal, both fec and hep domains will occur with equal probability. This explains the first term on the right-hand side of Eq. (4):

$$
\frac{f_{\mathrm{hcp}}+f_{\mathrm{fcc}}}{2}-f_{\mathrm{fcc}}=0.5 \Delta f_{\mathrm{hcp}-\mathrm{fcc}} \text {. }
$$

The minimum thickness of such a domain is three layers (because it takes three layers to distinguish $A B C$ from $A B A$ stacking). In a randomly stacked crystal, there is a 50\% probability that the first stacking fault will appear at the next layer. $25 \%$ for the following, and so on. It is easy to verify that the average domain thickness is four layers. The factor 0.25 in the second term on the right-hand side of Eq. (4) accounts for the fact that there is, on average, one stacking fault per four layers. The factor $s_{0}$ appears because we have to convert form free energy per unit area $\left(\gamma_{\mathrm{hcp}-\mathrm{fcc}}\right)$ to free energy per particle. Our simulations suggest that for small crystals (less than a thousand particles per plane) rhcp is more stable than fcc. Hence, only after the crystallites have grown beyond this size (corresponding to lateral dimensions of some ten microns for real colloidal crystals) can the slow annealing towards the stable phase commence. Let us, therefore, consider crystals that are sufficiently large, that we can ignore the stacking entropy. Then the driving force per particle to convert from rhcp to fcc is

$$
\Delta f_{\infty} \equiv 0.5 \Delta f_{\text {hcp-fcc }}+0.25 \gamma_{\text {hcp-fcc }} s_{0},
$$

which, in the present case, is approximately equal to $6 \cdot 10^{-4}$.

Let us now consider the growth of fcc crystallites at the expense of rhcp crystallites. It is plausible to assume that fcc crystallites grow where a [111]-grain boundary of the fcc crystal is in contact with an rhcp crystal. That is, we assume that the rate of restacking that occurs in the bulk is negligible. We also ignore shear-induced transformation from rhcp to fcc. ${ }^{16}$ In the case of colloidal crystal growth from solution, the velocity of the crystal front is, to a good approximation given by $3,4,7,8,15$

$$
\nu_{c r}=\frac{\zeta D}{\Lambda}\left(e^{\Delta \mu / k_{B} T}-1\right),
$$

where $\Delta \mu$ is the chemical-potential difference between liquid and solid. $D$, the (short-time) self-diffusion constant in the dense colloidal suspension, is typically one to two orders of magnitude smaller than the self-diffusion constant in a dilute suspension. $\Lambda$ is a characteristic distance over which a particle should diffuse in order to be incorporated in the crystal, and $\zeta$ is a factor of order unity. In order to arrive at an estimate for $\nu_{c r}$, we assume that grain boundaries are liquidlike, and the characteristic distance $\Lambda$ is of the order of the particle diameter $\sigma$. Moreover, we replace $\Delta \mu$ by $\Delta f_{\text {rhcp-fcc }}$. For a $200 \mathrm{~nm}$ colloid, a typical value for $D$ would be $D \approx 2 \cdot 10^{-10} \mathrm{~cm}^{2} \mathrm{~s}^{-1}$. The resulting estimate for $\nu_{c r}$ is $\nu_{c r} \approx 6 \cdot 10^{-9} \mathrm{~cm} \mathrm{~s}^{-1}$. Hence, this rough estimate suggests that it would take several months to grow a $1 \mathrm{~mm}$ fcc crystal, starting from a rhcp crystallite. It is, therefore, hardly surprising that only random stacking was observed in the microgravity experiments of Zhu et al. ${ }^{11}$ However, many studies of crystallization in colloidal suspensions last months or even years. The present analysis suggests that the fcc crystallites observed under those conditions could indeed be "true" hard-sphere crystal fcc phases. A second conclusion is that small crystallites (containing less than $30^{3} \approx 3 \cdot 10^{4}$ particles) will never become fcc-like (most likely, these will eventually "evaporate" due to Ostwald ripening). Intermediate-sized crystallites (containing less than $100^{3}$ $=10^{6}$ particles) can be fcc-like, but will always have an 
appreciable equilibrium concentration stacking faults. In practice, one would expect that a poly-crystalline sample will contain crystallites of many different size. As Eq. (4) shows, the driving force depends on the crystallite size. This is because $N_{\text {layer }}$, the number of particles per close-packed layer, is proportional to the cross section of the crystal. The smaller the linear dimensions of the crystal, the smaller the thermodynamic driving force to convert from rhcp to fcc. On basis of Eqs. (4) and (5), we expect the following size dependence of the rate at which crystallites convert from rhcp to fcc:

$$
\Gamma(\ell)=\nu_{c r}(\ell) / \ell=\left(\nu_{\infty}-c / \ell^{2}\right) / \ell,
$$

where $\ell$ is a measure for the linear dimension of a crystallite and the constant $c$ is given by

$$
c=\frac{\zeta D s_{0} \ln 2}{\Lambda} \text {. }
$$

The maximum conversion rate occurs for

$$
\ell_{m}=\sqrt{3 c / \nu_{\infty}}=\sqrt{\frac{3 \ln 2 s_{0}}{\Delta f_{\infty}}} .
$$

If we take a typical value of $200 \mathrm{~nm}$ for the diameter of hard-sphere colloids that are studied in experiments, this would correspond to a typical crystallite size of $12 \mu \mathrm{m}-$ in other words, crystallites of this size will be the first to convert to the fcc phase. This prediction could be tested using confocal scanning laser microscopy. In contrast, scattering experiments probe the total amount of solid that is transformed from rhcp to fcc, rather than number of crystallites. In this case, the measured rate of transformation will initially be dominated by the largest crystals. At longer times, the slower conversion of the smaller crystallites should show up. Recent light-scattering experiments by Dux and Versmold ${ }^{14}$ on charge-stabilized latex suspensions suggest that the conversion from random stacking to fcc follows a stretched ex- ponential decay. Stretched exponentials often arise as a superposition of simple exponentials with a wide distribution of time constants. However, as we do not know the crystallite-size distribution in these experiments, we cannot make a direct comparison between theory and the experimental data.

\section{ACKNOWLEDGMENTS}

The work of the FOM Institute is part of the research program of FOM and is made possible by financial support from the Netherlands organization for Scientific Research (NWO). We thank Alfons van Blaaderen and Willem Kegel for a critical reading of the manuscript.

${ }^{1}$ B. J. Alder and T. E. Wainwright, J. Chem. Phys. 27, 1208 (1957).

${ }^{2}$ W. W. Wood and J. D. Jacobson, J. Chem. Phys. 27, 1208 (1957).

${ }^{3}$ P. N. Pusey, in Liquids, Freezing and glass transition, edited by J. P. Hansen, D. Levesque, and J. Zinn-Justin (Elsevier, Amsterdam, 1991), pp. 763-942.

${ }^{4}$ W. B. Russel, Phase Transit. 21, 127 (1990).

${ }^{5}$ D. Frenkel and A. J. C. Ladd, J. Chem. Phys. 81, 3188 (1984).

${ }^{6}$ L. V. Woodcock, Nature (London) 385, 131 (1997).

${ }^{7}$ K. Schätzel and B. J. Ackerson, Phys. Rev. Lett. 68, 337 (1992).

${ }^{8}$ S. Henderson, Crystallization Mechanisms in Colloidal Systems, 1993, B. Sc. thesis, Royal Melbourne Institute of Technology.

${ }^{9}$ P. B. Bolhuis, D. Frenkel, S. Mau, and D. Huse, Nature (London) 388, 235 (1997).

${ }^{10}$ A. D. Bruce, N. B. Wilding, and G. J. Ackland, Phys. Rev. Lett. 79, 3002 (1997).

${ }^{11}$ J. Zhu, M. Li, R. Rogers, W. Meyer, R. H. Ottewill, W. B. Russel, and P. M. Chaikin, Nature (London) 387, 883 (1997).

${ }^{12}$ P. N. Pusey, W. van Megen, P. Bartlett, B. J. Ackerson, J. G. Rarity, and S. M. Underwood, Phys. Rev. Lett. 63, 2753 (1989).

${ }^{13}$ T. Palberg, Curr. Opin. Colloid Interface Sci. 2, 607 (1997).

${ }^{14}$ C. Dux and H. Versmold, Phys. Rev. Lett. 78, 1811 (1997).

${ }^{15}$ J. Frenkel, Kinetic Theory of Liquids (Oxford University Press, Oxford, 1946).

${ }^{16}$ S. E. Paulin, B. J. Ackerson, and M. S. Wolfe, Phys. Rev. E 55, 5812 (1997) 